\title{
The NapF protein of the Escherichia coli periplasmic nitrate reductase system: demonstration of a cytoplasmic location and interaction with the catalytic subunit, NapA
}

\author{
Correspondence \\ Jeffrey A. Cole \\ j.a.cole@bham.ac.uk
}

Received 24 May 2006

Revised 11 July 2006

Accepted 19 July 2006

\author{
Arjaree Nilavongse, ${ }^{1}$ T. Harma C. Brondijk, ${ }^{1}$ Tim W. Overton, ${ }^{1}$ \\ David J. Richardson, ${ }^{2}$ Emily R. Leach ${ }^{2}$ and Jeffrey A. Cole ${ }^{1}$ \\ ${ }^{1}$ School of Biosciences, University of Birmingham, Birmingham B15 2TT, UK \\ ${ }^{2}$ School of Biological Sciences, University of East Anglia, Norwich NR4 7TJ, UK
}

\begin{abstract}
The periplasmic nitrate reductase of Escherichia coli is important during anaerobic growth in lownitrate environments. The nap operon encoding this nitrate reductase comprises seven genes including a gene, nap $F$, that encodes a putative cytoplasmic iron-sulphur protein of uncertain subcellular location and function. In this study, $\mathrm{N}$-terminal sequence analysis, cell fractionation coupled with immunoblotting and construction of $\mathrm{LacZ}$ and PhoA fusion proteins were used together to establish that NapF is located in the E. coli cytoplasm. A bacterial two-hybrid protein-protein interaction system was used to demonstrate that NapF interacted in the cytoplasm with the terminal oxidoreductase NapA, but that it did not self-associate or interact with other electron-transport components of the $\mathrm{Nap}$ system, $\mathrm{NapC}, \mathrm{NapG}$ or $\mathrm{NapH}$, or with another cytoplasmic component, NapD. NapF, purified as a His 6 -tagged protein, exhibited spectral properties characteristic of an iron-sulphur protein. This protein was able to pull down NapA from soluble extracts of $E$. coli. A growth-based assay for NapF function in intact cell cultures was developed and applied to assess the effect of mutation of a number of conserved amino acids. It emerged that neither a highly conserved $\mathrm{N}$-terminal double-arginine motif, nor a conserved proline motif, is essential for NapF-dependent growth. The combined data indicate that NapF plays one or more currently unidentified roles in the post-translational modification of NapA prior to the export of folded NapA via the twin-arginine translocation pathway into the periplasm.
\end{abstract}

\section{INTRODUCTION}

The Escherichia coli genome encodes three distinct nitrate reductases. The membrane-bound nitrate reductases NarA and NarZ reduce nitrate in the cytoplasm (Berks et al., 1995a; Blasco et al., 1990; Iobbi-Nivol et al., 1990; Stewart, 1988). Nitrate reductase $\mathrm{A}$ is the predominant nitrate reductase when $E$. coli is grown under nitrate-sufficient conditions (Potter et al., 1999), whereas the poorly expressed nitrate reductase $\mathrm{Z}$ confers a selective advantage during the stationary phase or very slow growth (Iobbi et al., 1987, 1990; Clegg et al., 2006). Under nitrate-limiting conditions, a periplasmic nitrate reductase (Nap) is expressed (Potter et al., 1999; Wang et al., 1999; Stewart et al., 2002). Periplasmic nitrate reductases have been found in many different organisms, where they fulfil different physiological roles, depending on the species. For example, they can function as electron sinks during photosynthesis in

Abbreviations: MK, menaquinone; TAT, twin-arginine translocase; UQ, ubiquinone; X-P, 5-bromo-4-chloro-3-indolyl phosphate.
Rhodobacter species (Reyes et al., 1996, 1998) and during aerobic growth of Paracoccus species on highly reduced carbon sources (Richardson \& Ferguson, 1992; Sears et al., 2000), or they may be used during anaerobic respiration, as in E. coli (Potter \& Cole, 1999; Brondijk et al., 2002; Stewart et al., 2002). In spite of their different roles, there are several similarities between the periplasmic nitrate reductase systems of different species. Many have four proteins in common: NapA, NapB, NapC (or a homologue) and NapD. NapA is a periplasmic protein that binds a molybdenum bis-molybdopterin (Mo-bis-MGD) cofactor and a $4 \mathrm{Fe} 4 \mathrm{~S}$ cluster. The cofactors are incorporated into the enzyme in the cytoplasm and it is then exported as a folded protein via the twin-arginine translocase (TAT) system. NapB is a di-haem periplasmic protein that is exported by the Sec pathway. NapA and NapB then interact in the periplasm, with the site of nitrate reduction on the NapA Mo-bis-MGD cofactor and NapB functioning as an electron donor to NapA. NapC is a tetra-haem cytochrome that is anchored into the cytoplasmic membrane and is believed to be the quinol dehydrogenase of the system (Berks et al., 
1995a; Simon et al., 2003; Brondijk et al., 2004). NapD is located in the cytoplasm (Berks et al., 1995a) and may play a role in NapA maturation prior to export (Potter \& Cole, 1999).

In addition to these four proteins, many nap operons also encode additional proteins, mostly of unknown function. For example, in E. coli Nap is encoded by the napFDAGHBC operon. Based on their primary structure, NapF, NapG and $\mathrm{NapH}$ are all believed to be non-haem iron-sulphur proteins. We recently reported that $\mathrm{NapH}$ is an integral membrane protein with four membrane-spanning helices (Brondijk et al., 2004). The N- and C-termini, as well as the two iron-sulphur centres, are located in the cytoplasm. NapG is loosely attached on the periplasmic side of the membrane, and is transported across the membrane by the TAT pathway (Brondijk et al., 2004; Santini et al., 1998; Weiner et al., 1998; Berks et al., 2000). NapG and NapH are both required for optimal electron transfer from ubiquinol to the periplasmic Nap complex (Brondijk et al., 2004). The location of NapF has been the subject of debate, with early contradictory schemes placing it in either the periplasm (Berks et al., 1995a) or the cytoplasm (Reyes et al., 1998). This has been because its N-terminal sequence includes two arginine residues in a motif with some similarity to a TAT export signal (Weiner et al., 1998; Berks et al., 2000) that suggests a periplasmic location (Reyes et al., 1998). However, NapF lacks other N-terminal features typical of proteins secreted via the TAT pathway, which points to a cytoplasmic location (Berks et al., 1995a). Only two napF mutants have so far been characterized, one in Rhodobacter sphaeroides, in which NapF is essential for nitrate reduction (Reyes et al., 1998), the other in E. coli, in which loss of $\mathrm{NapF}$ function results in subtle nitrate-dependent growth defects, especially during adaptation from aerobic to anaerobic growth (Brondijk et al., 2002). Experimental evidence has recently emerged for the $R$. sphaeroides NapF that supports a cytoplasmic location (Olmo-Mira et al., 2004), but further data from other organisms is required to establish whether this is also true for other bacteria. This is provided in the current work, which presents evidence that $\mathrm{NapF}$ is indeed located in the cytoplasm and provides the first in vivo evidence that it can interact with NapA in this subcellular compartment. Since mature NapA is located in the periplasm, this points to a role for NapF in interaction with NapA during the maturation of the enzyme in the cytoplasm prior to export of the mature form into the periplasm.

\section{METHODS}

Strains, plasmids and growth conditions. Strains of Escherichia coli K-12 and plasmids used or constructed during this study are listed in Table 1. Strains JCB4141 and 4142 lack both of the cytoplasmic nitrate reductases, $\mathrm{A}$ and $\mathrm{Z}$, and, due to the narL mutation, overexpress the periplasmic nitrate reductase during anaerobic growth in the presence of nitrate. Anaerobic growth of these strains in minimal medium in the presence of a non-fermentable carbon source such as glycerol requires a reducible electron acceptor, so growth with nitrate is Nap-dependent (Brondijk et al., 2002). Bacteria were grown aerobically in LB ( $10 \mathrm{~g}$ tryptone $1^{-1}, 5 \mathrm{~g}$ yeast extract $\mathrm{l}^{-1}$ and $5 \mathrm{~g} \mathrm{NaCl}^{-1}$ ) or anaerobically in MS medium $[4 \cdot 5 \mathrm{~g}$ $\mathrm{KH}_{2} \mathrm{PO}_{4} \mathrm{l}^{-1}, 10 \cdot 5 \mathrm{~g} \mathrm{~K}_{2} \mathrm{HPO}_{4} \mathrm{l}^{-1}, 1 \mathrm{~g} \mathrm{NH}_{4} \mathrm{SO}_{4} \mathrm{l}^{-1}, 0 \cdot 05 \mathrm{~g} \mathrm{MgCl}_{2}$ $1^{-1}, 2 \cdot 5 \mathrm{~g}$ nutrient broth (NB) base $1^{-1}, 10 \mu \mathrm{M}$ sodium molybdate, $10 \mu \mathrm{M}$ sodium selenate and $1 \mathrm{ml}^{-1}$ of $E$. coli sulphur-free salts (Pope \& Cole, 1984) supplemented with $20 \mathrm{mM} \mathrm{NaNO}_{3}$ and $0 \cdot 4 \%$ $(\mathrm{v} / \mathrm{v})$ glycerol or $0.4 \%(\mathrm{w} / \mathrm{v}) \mathrm{D}$-glucose]. When appropriate $100 \mu \mathrm{g}$ ampicillin $\mathrm{ml}^{-1}$ and/or $50 \mu \mathrm{g}$ kanamycin $\mathrm{ml}^{-1}$ were added. Unless stated otherwise, cultures were grown at $37^{\circ} \mathrm{C}$.

Construction of the pAN100 series of plasmids. Primers used for cloning steps are listed in Table 2. The cloning vector for the pAN100 series of plasmids was pBR322 (Bolivar et al., 1977). The DNA sequence of the napF promoter and coding region was amplified by PCR using plasmid pJG460 (Grove et al., 1996) as a template. Two primers, pNapFEcoRI and NapFBamH2, containing $E c o$ RI and BamHI at the end of each primer, respectively, were used to create the $900 \mathrm{bp}$ DNA fragment. The PCR fragment was digested with EcoRI and BamHI and then ligated into the EcoRI-BamHI site of pBR322. The resulting plasmid, pAN101, contains the complete DNA sequence of napF, expressed under the control of the nap promoter.

Two highly conserved sequence motifs in the NapF protein family are a double-arginine motif close to the $\mathrm{N}$-terminus, and the unusual sequence I-R-P-P-W 12-20 residues downstream. Both motifs are also present in NapG, which was previously shown to be exported into the periplasm (Brondijk et al., 2004). As highly conserved sequences are often essential for NapF function, the double-arginine motif in the putative signal sequence of NapF was changed to a double alanine using the Quikchange Site-Directed Mutagenesis kit (Stratagene). First a megaprimer was generated by PCR using primer NapF BamH2 and the mutagenic primer NapF RR to AA with pJG600 as template. The purified $500 \mathrm{bp}$ product was then used in a PCR reaction with primer pNapF EcoRI and pJG600 as template. The $900 \mathrm{bp}$ product was cloned into pBR322 to yield plasmid pAN103. Similarly, the proline motif in NapF was modified using the primers listed in Table 2 to generate the derivatives pAN111, encoding the substitution P24A, pAN112 (P25A), pAN113 (W26Y) and pAN114, encoding all three substitutions (P24A P25A W26Y).

Construction of the pLP800 series of plasmids. Plasmid pLP802 was constructed by amplifying a DNA fragment containing napF using primers NapFNdeI, which created an NdeI restriction site upstream from the start codon of napF, and NapFBamH2, which created a BamHI restriction site at the end of napF. The PCR fragment was then restricted with $\mathrm{NdeI}$ and BamHI and cloned into pET11c vector. Plasmid pLP802 expresses NapF under the control of an inducible T7 promoter. To create plasmid pLP804, which expresses napF with a myc epitope, the napF gene was amplified using primers NapFNdeI and NapFmycBamHI. The latter primer creates a Myc tag at the C-terminus of $\mathrm{NapF}$ and BamHI restriction site immediately after the stop codon. The Ndel/BamHI-digested fragment was then cloned into pET11c. Plasmid pLP805 was constructed to contain the napF gene with a mutation at the putative twin-arginine motif. The mutagenic primer NapFRRtoAA was designed to contain the sequences that encoded two alanines instead of two arginines at the conserved position and an NdeI site was created. The mutated napF gene was amplified using primers NapFRRtoAA and NapFmycBamHI and subsequently cloned into pET11c (as described above). Plasmid pLP805 expresses napF with mutations at the conserved twin-arginine motif and a myc tag from the $\mathrm{T} 7$ promoter.

Construction of plasmids and detection of PhoA and LacZ fusion proteins. The napF gene, including its promoter but without the translational termination codon, was amplified by PCR 
Table 1. E. coli strains and plasmids

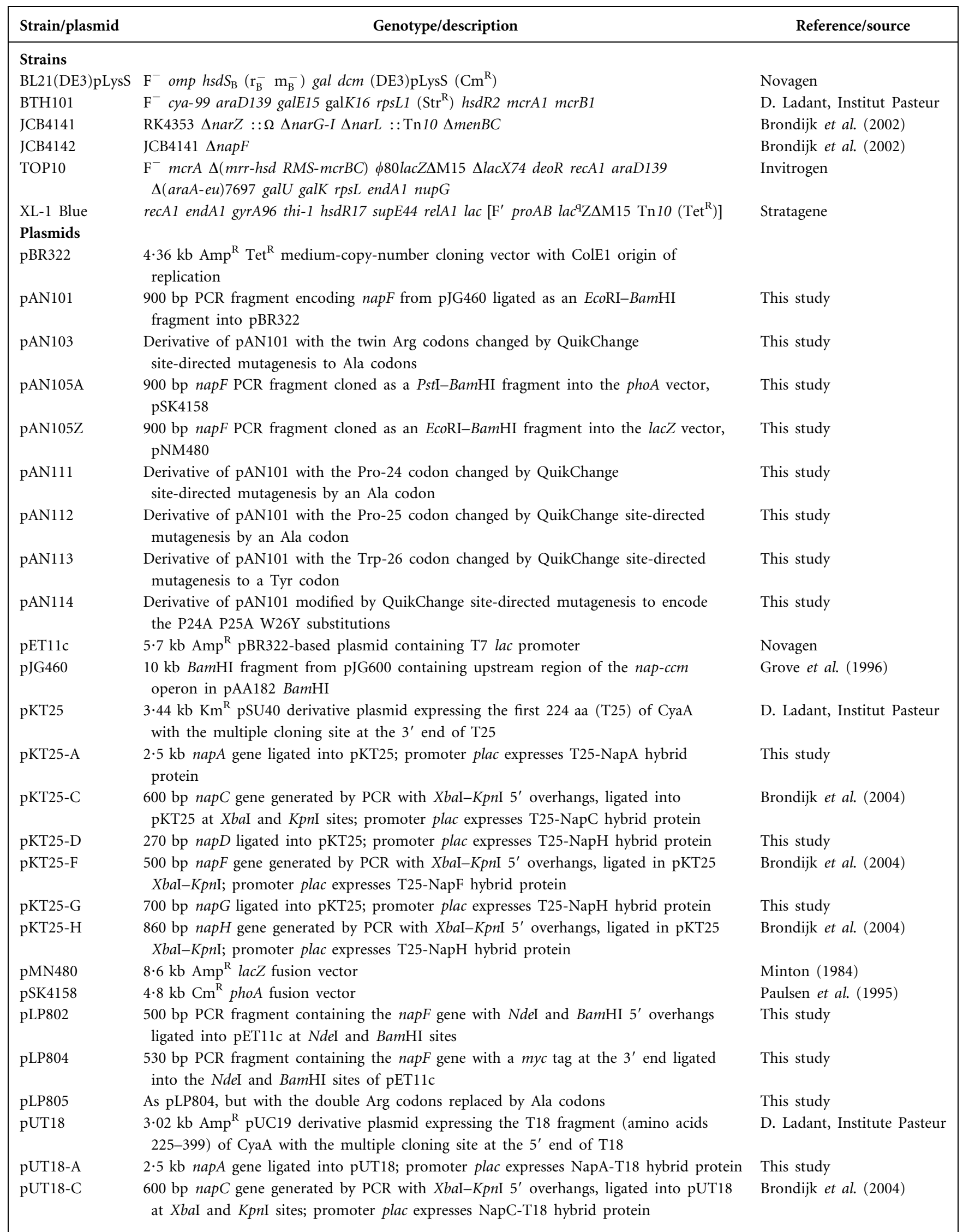


Table 1. cont.

\begin{tabular}{|c|c|c|}
\hline Strain/plasmid & Genotype/description & Reference/source \\
\hline pUT18-D & 270 bp napD gene ligated into pUT18; promoter plac expresses NapD-T18 hybrid protein & This study \\
\hline pUT18-F & $\begin{array}{l}500 \text { bp napF gene generated by PCR with } X b a \mathrm{I}-K p n \mathrm{I} 5^{\prime} \text { overhangs, ligated in pUT18 } \\
\text { XbaI-KpnI; promoter plac expresses NapF-T18 hybrid protein }\end{array}$ & Brondijk et al. (2004) \\
\hline pUT18-G & 700 bp napG gene ligated into pUT18; promoter plac expresses NapG-T18 hybrid protein & This study \\
\hline pUT18-H & $\begin{array}{l}860 \text { bp napH gene generated by PCR with } X b a \mathrm{I}-K p n \mathrm{I} 5^{\prime} \text { overhangs, ligated in pUT18 } \\
\text { XbaI-KpnI; promoter plac expresses NapH-T18 hybrid protein }\end{array}$ & Brondijk et al. (2004) \\
\hline pUT18C & $\begin{array}{l}3.02 \mathrm{~kb} \mathrm{Amp} \mathrm{R}^{\mathrm{R}} \text { pUC19 derivative plasmid expressing the T18 fragment (amino acids } \\
225-399 \text { ) of CyaA with the multiple cloning site at the } 3^{\prime} \text { end of T18 }\end{array}$ & D. Ladant, Institut Pasteur \\
\hline pUT18C-C & $\begin{array}{l}600 \mathrm{bp} n a p G \text { gene generated by PCR with } X b a \mathrm{I}-K p n \mathrm{I} 5^{\prime} \text { overhangs, ligated into pUT18C } \\
\text { at XbaI and } K p n \mathrm{I} \text { sites; promoter plac expresses T18-NapC hybrid protein }\end{array}$ & Brondijk et al. (2004) \\
\hline pUT18C-A & $\begin{array}{l}2 \cdot 5 \mathrm{~kb} \text { napA gene generated by PCR ligated into pUT18C; promoter plac expresses } \\
\text { T18-NapA hybrid protein }\end{array}$ & This study \\
\hline pUT18C-D & $\begin{array}{l}270 \text { bp napD gene generated by PCR ligated into pUT18C; promoter plac expresses } \\
\text { T18-NapD hybrid protein }\end{array}$ & This study \\
\hline pUT18C-F & $\begin{array}{l}500 \text { bp napF gene generated by PCR with } X b a \mathrm{I}-K p n \mathrm{I} 5^{\prime} \text { overhangs, ligated in pUT18C } \\
\text { XbaI-KpnI; promoter plac expresses T18-NapF hybrid protein }\end{array}$ & Brondijk et al. (2004) \\
\hline pUT18C-G & $\begin{array}{l}700 \text { bp napG gene generated by PCR ligated into pUT18C at XbaI and KpnI sites; } \\
\text { promoter plac expresses T18-NapC hybrid protein }\end{array}$ & This study \\
\hline pUT18C-H & 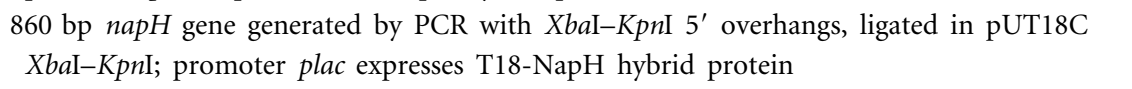 & Brondijk et al. (2004) \\
\hline pEL6HisNapF & napF excised from pLP802 and cloned into the $\mathrm{His}_{6}$-tag vector pET16B & This study \\
\hline
\end{tabular}

with primers that introduced a PstI or EcoRI site upstream of the nap promoter and a Bam HI site at the fusion point (Table 2). The PstI-BamHI fragments were cloned into pSK4158 (Manoil, 1991) to create in-frame phoA fusions and the EcoRI-BamHI fragments into pNM480 (Minton, 1984) to create in-frame lacZ fusions. Transformants were plated onto selective LB agar with $40 \mu \mathrm{g}$ 5-bromo-4chloro-3-indolyl phosphate (X-P) $\mathrm{ml}^{-1}$ or $40 \mu \mathrm{g} \mathrm{X}-\mathrm{Gal} \mathrm{ml}{ }^{-1}$. PhoA and $\beta$-galactosidase activities were measured as described previously (Karimova et al., 1998; Ladant \& Ullmann, 1999).

Preparation of periplasmic, cytoplasmic and membrane extracts from bacterial cultures. Cultures were grown aerobically in 11 of LB and induced with $0.5 \mathrm{mM}$ IPTG at $\mathrm{OD}_{650} \approx 0.5$. After $4 \mathrm{~h}$ of induction, bacteria were harvested, washed with $50 \mathrm{mM}$ Tris/ $\mathrm{HCl}$ buffer $\mathrm{pH} 8$ and resuspended in $15 \mathrm{ml}$ of a freshly prepared solution of SET buffer $(0.5 \mathrm{M}$ sucrose, $5 \mathrm{mM}$ EDTA and $50 \mathrm{mM}$ Tris/ $\mathrm{HCl}, \mathrm{pH} 8 \cdot 0$ ) containing $600 \mu \mathrm{g}$ lysozyme $\mathrm{ml}^{-1}$ (Brondijk et al., 2004). The resuspended pellet was incubated at $30^{\circ} \mathrm{C}$ for $1 \mathrm{~h}$ to allow the release of periplasmic proteins. The cell suspension was then centrifuged at $12000 \mathrm{~g}$ for $30 \mathrm{~min}$ to pellet the spheroplasts. The periplasmic fraction was dialysed against $1 \mathrm{mM}$ Tris/ $\mathrm{HCl}$, $\mathrm{pH} 8 \cdot 0$, freeze-dried and resuspended in sterile distilled water. The spheroplasts were resuspended in $5 \mathrm{ml} 50 \mathrm{mM}$ Tris/ $\mathrm{HCl} \mathrm{pH} 8.0$ and passed through a French pressure cell at 10000 p.s.i. The slurry, consisting of unbroken bacteria, cell membranes and cytoplasm, was centrifuged at $5000 \mathrm{~g}$ for $10 \mathrm{~min}$ to sediment unbroken bacteria and inclusion bodies, and then at $170000 \mathrm{~g}$ for $1 \mathrm{~h}$ to sediment the membrane fraction. Soluble cytoplasmic proteins in the supernatant were removed. The resulting membrane pellet was homogenized with $5 \mathrm{ml} 50 \mathrm{mM}$ Tris/ $\mathrm{HCl} \mathrm{pH} 8.0$ and further purified on a $36-54 \%(w / v)$ sucrose gradient to ensure that it was free from unbroken bacteria, outer membranes and inclusion bodies.

Purification of $\mathrm{His}_{6}-\mathrm{NapF}$ and demonstration of the pulldown of NapA from soluble fractions of $E$. coli. One-litre batches of E. coli BL21(DE3)pLysS containing the His ${ }_{6}$-NapF expression vector pELHisNapF were grown up aerobically on LB medium at $37^{\circ} \mathrm{C}$. When the $\mathrm{OD}_{600}$ reached 0.6 the cells were induced with $0.5 \mathrm{mM}$ IPTG and the cultures incubated at $25^{\circ} \mathrm{C}$ for a further $4 \mathrm{~h}$. The cells were then harvested by centrifugation and a soluble extract was prepared by French pressure cell treatment of the cell pellet followed by resuspension in $20 \mathrm{mM}$ Tris/ $\mathrm{HCl}$ buffer $\mathrm{pH} 7 \cdot 5$. Purification of the $\mathrm{His}_{6}-\mathrm{NapF}$ protein then involved sequential application of Ni-affinity, DEAE anion-exchange and S200 gel-exclusion chromatographies, followed by a second $\mathrm{Ni}$-affinity step. The DEAE column was developed with a $0-500 \mathrm{mM} \mathrm{NaCl}$ gradient and $\mathrm{NapF}$ eluted at around $300 \mathrm{mM} \mathrm{NaCl}$. The Ni-affinity columns were developed with 10-500 mM imidazole gradients and $\mathrm{His}_{6}-\mathrm{NapF}$ eluted at around $480 \mathrm{mM}$ imidazole. In all cases NapF-containing fractions were detected by SDS-PAGE analysis of column fractions.

To demonstrate pull-down of NapA by NapF, soluble fractions were prepared from E. coli grown anaerobically on a glycerol/nitrate minimal medium and applied to Ni-affinity columns that were either charged with $\mathrm{His}_{6}-\mathrm{NapF}$ or left uncharged. Columns were washed with $20 \mathrm{mM}$ Tris/ $\mathrm{HCl}$ and $10 \mathrm{mM}$ imidazole buffer $\mathrm{pH} 7 \cdot 5$, and then a $10-500 \mathrm{mM}$ imidazole gradient in $20 \mathrm{mM}$ Tris/ $\mathrm{HCl}$ buffer $\mathrm{pH} 7 \cdot 5$ was applied in a total volume of $60 \mathrm{ml}$. Column eluate fractions were subjected to SDS-PAGE and stained with Coomassie brilliant blue or Western blotted and then probed with anti-NapA antibodies.

SDS-PAGE, Western blotting and $\mathbf{N}$-terminal sequencing. For SDS-PAGE, a $15 \%(\mathrm{w} / \mathrm{v})$ resolving gel and a $6 \%(\mathrm{w} / \mathrm{v})$ stacking polyacrylamide gel in an $18 \mathrm{~cm}$ square-slab apparatus were used exactly as described previously (Brondijk et al., 2004). For wholecell samples, the bacterial pellet was resuspended in sample buffer containing $12 \%(\mathrm{v} / \mathrm{v}) 2$-mercaptoethanol and heated at $95^{\circ} \mathrm{C}$ for $10 \mathrm{~min}$ prior to loading. Protein bands were stained with Coomassie brilliant blue or transferred to a PVDF membrane for Western blot 
Table 2. Oligonucleotide primers

Key: +, coding strand; - complementary strand. Incorporated restriction sites are underlined; bases changed by site-directed mutagenesis are shown in bold; the myc tag sequence is shown in italic.

\begin{tabular}{|c|c|c|c|}
\hline Primer name & Strand & $\begin{array}{l}\text { Restriction } \\
\text { site }\end{array}$ & Sequence $\left(5^{\prime}-3^{\prime}\right)$ \\
\hline NapFNdeI & + & NdeI & TATTCCCATATGAAGATTGATGCATCCCG \\
\hline NapF myc BamHI & - & BamHI & $\begin{array}{l}\text { ATAATAGGATCCTTACAGATCCTCTTCTGAGATGAGTTTT- } \\
\text { TGTTCGTGTGCATGGAGATACTCCG }\end{array}$ \\
\hline pNapFEcoRI & + & EcoRI & GAACGAATTCCCGATGATGTCGCTG \\
\hline NapF(P1toA)UP & + & - & GGTATCCGTGCGCCCTGGTCCGG \\
\hline NapF(P1toA)DOWN & - & - & GGACCAGGGCGCACGGATACCG \\
\hline $\operatorname{NapF}(\mathrm{P} 2$ toA $) \mathrm{UP}$ & + & - & ATCCGTCCGGCGTGGTCCGGTG \\
\hline NapF(P2toA)DOWN & - & - & ACCGGACCACGCCGGACGGATACCG \\
\hline \multicolumn{4}{|c|}{ Two-hybrid interaction primers } \\
\hline NapAXbaI & + & $X b a \mathrm{I}$ & TATATTCTAGAGATGAAACTCAGTCGTCGTAGC \\
\hline NapAKpnI & - & $K p n \mathrm{I}$ & ATAAGGTACCCGCACCTTCTCCAGTTTGACCGC \\
\hline NapAKpnIstop & - & $K p n \mathrm{I}$ & ATAAGGTACCCGTTACACCTTCTCCAGTTTGAC \\
\hline NapDXbaI & + & $X b a \mathrm{I}$ & TATATTCTAGAGATGCACACTAACTGGCAAG \\
\hline NapDKpnI & - & KpnI & ATAAGGTACCCGTGGTGTTTCCTCACCTTGCTC \\
\hline NapDKpnIstop & - & KpnI & ATAAGGTACCCGTCATGGTGTTTCCTCACCTTGC \\
\hline NapGXbaI & + & $X b a \mathrm{I}$ & TATTATACTCTAGAGATGTCCCGGTCAGCGAAACC \\
\hline NapGKpnI & - & $K p n \mathrm{I}$ & ATAATAAGGTACCCGCGATTTGCCATTGTTCCC \\
\hline NapGKpnIstop & - & KpnI & ATAATAAGGTACCCGTCATTACGATTTGCCATTGTTCCC \\
\hline \multicolumn{4}{|l|}{ phoA-lacZ fusion primers } \\
\hline
\end{tabular}

analysis. Anti-Myc antibody was purchased from Invitrogen and anti-mouse IgG was obtained from TROPIX. The antibodies were detected using a chemiluminescent detection system (TROPIX). For the $\mathrm{N}$-terminal sequencing, proteins were transferred on to PVDF membrane and stained with Coomassie brilliant blue. Bands of interest were excised and used directly for automated degradation sequencing by Alta Bioscience (University of Birmingham).

Bacterial two-hybrid system. The coding sequences of each of the nap genes (napF, napD, napA, napG, napH and napC), with or without the stop codon, as appropriate, were cloned in-frame into pUT18, pUT18C and pKT25. These plasmids encode fusion proteins with the T18 and T25 fragments of Bordetella pertussis adenylate cyclase (Karimova et al., 1998, 2000; Ladant \& Ullmann, 1999). Two complementary plasmids were transformed into the adenylatecyclase-deficient (cya) E. coli strain BTH101. Transformants were plated on MacConkey agar supplemented with $1 \%$ maltose and incubated at $30^{\circ} \mathrm{C}$. Development of red colonies, indicative of a positive interaction, was followed for $72 \mathrm{~h}$. The strength of the interactions was determined by measuring the $\beta$-galactosidase activity of the transformants after anaerobic growth to an $\mathrm{OD}_{650}$ of 0.5 in $\mathrm{LB}$ supplemented with $20 \mathrm{mM} \mathrm{NaNO}_{3}$ and $0 \cdot 4 \%$ glycerol.

Rates of nitrate reduction by physiological substrates and reduced methylviologen. A nitrate electrode was used to measure rates of nitrate reduction by washed bacterial suspensions incubated with $1.2 \mathrm{mM}$ nitrate and the physiological substrates glucose, glycerol or formate (Pope \& Cole, 1984). Nitrate reductase activities of permeabilized bacteria incubated with $1 \mathrm{mM}$ nitrate and reduced methylviologen were determined as described previously (Potter \& Cole, 1999).

Chemical assays. Aerobic and anaerobic cultures were grown and assayed for $\beta$-galactosidase activity as described previously (Jayaraman et al., 1987). Nitrite concentrations were measured according to Pope \& Cole (1984). Protein concentrations were determined by the Lowry method.

\section{RESULTS}

\section{The subcellular localization of NapF}

To initiate investigation of the subcellular location of $\mathrm{NapF}$, the complete coding sequence for napF was fused in-frame to phoA in plasmid pSK4158, and to lacZY in plasmid pNM480, to generate plasmids pAN105A and pAN105Z, respectively (Table 1). The fusion plasmids were transformed into E. coli TOP10, and transformants were 
plated onto nutrient agar supplemented with chromogenic substrates, either X-P or X-Gal. Both of the untransformed control strains, as well as bacteria transformed with the phoA fusion, pAN105A, formed white colonies on X-P plates (Table 3 ). In contrast, although the untransformed TOP10 formed white colonies on X-Gal, bacteria transformed with pAN105Z formed blue colonies. After anaerobic growth in LB supplemented with glycerol, nitrate and appropriate antibiotics, no alkaline phosphatase activity was detected in the pAN105A transformant, but the $\beta$ galactosidase activity of the pAN105Z transformant was $820 \mathrm{nmol}$ ONPG hydrolysed $\min ^{-1}$ (mg dry mass) ${ }^{-1}$ compared with 85 units in the untransformed control (Table 3). Since $\beta$-galactosidase is regarded as a marker for cytoplasmic enzymes and alkaline phosphatase is regarded as a marker of periplasmic enzymes these data support the view that NapF is located in the cytoplasm.

The use of gene fusions alone can give ambiguous results on subcellular location of TAT-dependent enzymes since PhoA is a poor substrate for the TAT (Stanley et al., 2002). Consequently the subcellular location of NapF was also assessed using antibody-based approaches. To facilitate this, E. coli BL21(DE3)pLysS derivatives harbouring the plasmids pLP802 and pAN804, encoding NapF and a NapF-Myc fusion protein, respectively, were constructed (Table 1). To avoid disruption of any signal peptide that might be required for export to the periplasm, NapF was tagged with the Myc epitope at its C-terminus. This tagging approach was used to demonstrate the periplasmic location of the TAT-dependent NapG protein (Brondijk et al., 2004). Cultures were initially grown aerobically at $37^{\circ} \mathrm{C}$ in $\mathrm{LB}$ medium and induced at an $\mathrm{OD}_{650}$ of 0.5 by the addition of $0.5 \mathrm{mM}$ IPTG. Proteins in samples removed at intervals of $1 \mathrm{~h}$ were separated by SDS-PAGE. An intense $18 \mathrm{kDa}$ band corresponding to NapF was detected 2 or $3 \mathrm{~h}$ after induction, but not before induction, in the bacteria transformed with pLP802 (Fig. 1a). This was replaced by a $20 \mathrm{kDa}$ band in bacteria transformed with pLP804 (Fig. 1a). This $2 \mathrm{kDa}$ shift in molecular mass was expected, as a result of the presence of the Myc epitope. N-terminal sequencing of the excised bands established the sequence
Met-Lys-Ile-Asp-Ala, which corresponds to the primary translation product without cleavage of an $\mathrm{N}$-terminal leader sequence. This confirmed that these bands were indeed NapF and NapF-Myc and that the protein produced from pLP804 was suitable for use in further analyses in subcellular fractionation experiments.

For these fractionation experiments, cultures were grown at $25^{\circ} \mathrm{C}$ in anaerobic, minimal salts medium supplemented with glucose and fumarate, a condition that minimized formation of inclusion bodies. After overnight growth in 21 medium, NapF-Myc synthesis was induced by the addition of $0.5 \mathrm{mM}$ IPTG; the bacteria were harvested after a further $4 \mathrm{~h}$ and fractionated into periplasm, cytoplasm and cytoplasmic membrane fractions. After SDS-PAGE and Western blotting of equal amounts of protein in each fraction, NapF-Myc was clearly detected in the cytoplasm and membrane fractions of induced bacteria (Fig. 1b). No NapF-Myc was detected in the periplasmic fraction (Fig. 1b). NapF-Myc in the cytoplasm and membrane fractions migrated with the same apparent mass of $20 \mathrm{kDa}$ as the uncleaved, full-length NapF-Myc. NapF in the membrane fraction could be readily solubilized by treatment of the membranes with $0 \cdot 1 \%$ Triton X-100, $6 \mathrm{M}$ urea or $0 \cdot 2 \%$ sodium deoxycholate and retained the same apparent mass of $20 \mathrm{kDa}$ that was characteristic of the water-soluble cytoplasmic material. These subcellular localization experiments are fully consistent with the results of the gene fusion experiments and together the two approaches provide unequivocal evidence that $\mathrm{NapF}$ is a cytoplasmic protein loosely attached to the inner side of the membrane.

It is notable that NapF shares a high degree of sequence identity with NapG, which we have previously shown has a functional TAT signal sequence that targets it to the periplasm (Brondijk et al., 2004). Primary structure analysis of E. coli NapF reveals the sequence SRRGILT close to the Nterminus, which is similar to the sequence SRRxFLK that is typical of leader peptides of proteins secreted into the periplasm by the TAT pathway. Multiple sequence analysis of NapF proteins from a range of proteobacteria reveal that this twin-arginine sequence is highly conserved, suggesting

Table 3. Enzyme activities of NapF-PhoA/LacZ fusions

Enzyme activities are nmol X-Gal or X-P hydrolysed $\min ^{-1}$ (mg dry weight $)^{-1}$. The data are means \pm SD $(n=3)$.

\begin{tabular}{|lcc|}
\hline Plasmid & $\begin{array}{c}\text { Colour on X-Gal or } \\
\text { X-P indicator plates }\end{array}$ & $\begin{array}{c}\boldsymbol{\beta} \text {-Galactosidase or alkaline } \\
\text { phosphatase activity }\end{array}$ \\
\hline LacZ fusion & White & $85( \pm 2)$ \\
pNM480 (No fusion) & Blue & $820( \pm 170)$ \\
pAN105Z (NapF-LacZ) & White & $4( \pm 1)$ \\
PhoA fusion & White & $5( \pm 1)$ \\
pSK4158 (No fusion) & & \\
pAN105A (NapF-PhoA) & & \\
\hline
\end{tabular}



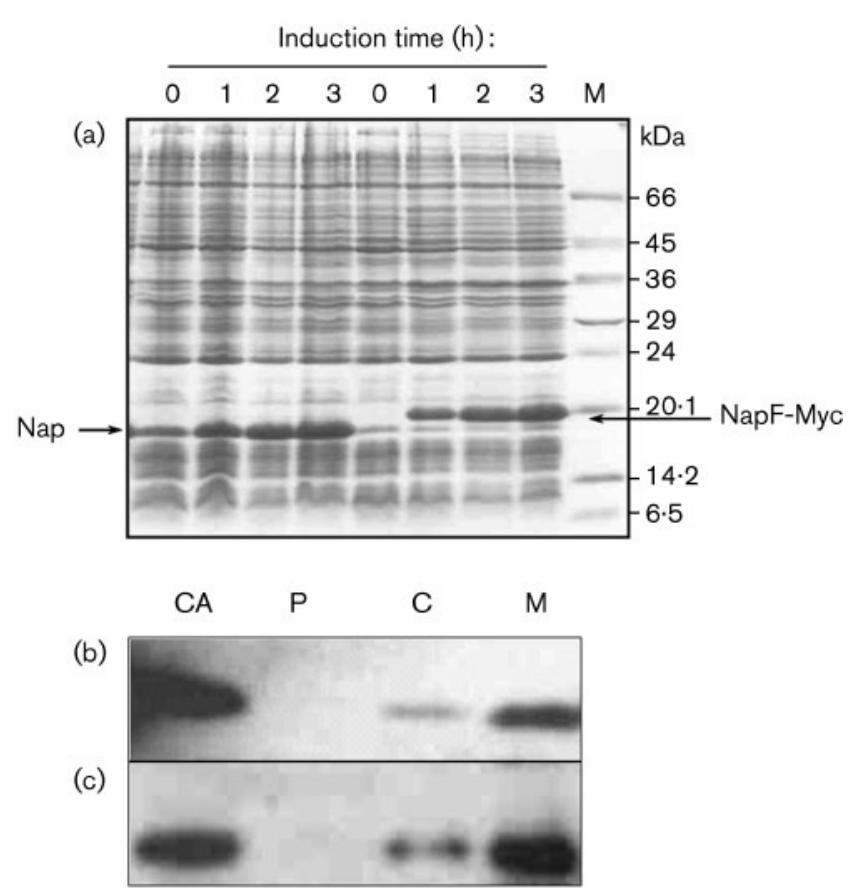

Fig. 1. Overexpression of $\mathrm{NapF}$ and NapF-Myc in E. coli BL21(DE3)pLysS and localization of the NapF and NapF R7A : R8A proteins. (a) SDS-PAGE analysis and staining by Coomassie brilliant blue of whole-cell samples. Cultures of $E$. coli BL21(DE3)pLysS/pLP802 (NapF) and E. coli BL21(DE3)pLysS/pLP804 (NapF-Myc) were induced with IPTG and grown aerobically for $3 \mathrm{~h}$ at $37^{\circ} \mathrm{C}$. The whole-cell samples were lysed and separated on a $15 \%$ SDS-polyacrylamide gel. (b, c) Cultures of $E$. coli BL21(DE3)pLysS/pLP804 expressing wild-type NapF (b) and BL21(DE3)pLysS/pLP805 expressing NapF R7A:R8A (c) were grown anaerobically at $25^{\circ} \mathrm{C}$ and induced with IPTG. After $4 \mathrm{~h}$ incubation, cells were harvested and fractionated into periplasm, cytoplasm and membrane. The following samples were separated by $15 \%$ SDS-PAGE and analysed by Western blotting using anti-Myc antibodies and enhanced chemiluminescence: $\mathrm{CA}$, whole cells after induction; $\mathrm{P}$, periplasm (50 $\mu \mathrm{g}$ protein); $\mathrm{C}$, cytoplasm (50 $\mu \mathrm{g}$ protein); $\mathrm{M}$, membrane fraction after French pressing and purification on a sucrose density gradient ( $50 \mu \mathrm{g}$ protein).

that it has functional significance. However, a hydrophobic region typical of TAT-dependent leader peptides does not follow it. Since the experiments described above establish that NapF is a cytoplasmic protein, the 'RR' motif conserved in many NapF proteins might simply be a remnant of the TAT motif that is not essential for NapF function. This was investigated by changing the codons for the doublearginine motif to alanine codons by site-directed mutagenesis. To develop an in vivo assay for NapF function we took advantage of our earlier observation that NapF is essential for nitrate-dependent growth of an E. coli strain defective in naphthoquinone synthesis. This strain, JCB4141, is defective in nitrate reductase $\mathrm{A}$, nitrate reductase $\mathrm{Z}$ and menaquinone (MK) synthesis. Strain JCB4141 adapted slowly when transferred from aerobically grown LB pre-cultures to an anaerobic minimal medium with glycerol as the sole carbon source and $20 \mathrm{mM}$ nitrate as the only terminal electron acceptor (Fig. 2a). This growth exhibited a clear biphasic character, with a slow first phase $(0-10 \mathrm{~h})$ and faster second phase $(10-20 \mathrm{~h})$. This slow phase was not apparent when the adapted cultures were further subcultured into the same anaerobic growth conditions. The growth was totally dependent upon the addition of nitrate. The napF derivative strain JCB4142 consistently exhibited a low rate of growth after transfer from the aerobic to anaerobic culture conditions but could not adapt to the new growth conditions and enter into the second fast growth phase (Fig. 2a), suggesting that NapF was needed to facilitate this adaptation. The second growth phase of JCB4142 was fully restored by transformation with plasmid pAN101, in which napF is cloned into pBR322 (Fig. 2b). This then established a functional growth-based assay for active NapF.

The modified napF gene encoding NapF in which the RR motif was substituted by AA was amplified by PCR and cloned into pBR322 to generate plasmid pAN103 (Table 1). This plasmid was as effective as pAN101 in complementing the growth defect of strain JCB4142, establishing that the arginine motif is not essential for NapF function (Fig. 2b). These data also further established that NapF is not targeted to the periplasm by the TAT secretion system, as it would not have been expected to complement growth had it been targeted to the wrong subcellular compartment.

Plasmid pAN101 was also used as the template to introduce point mutations into a conserved $\mathrm{R}-\mathrm{P}-\mathrm{P}-\mathrm{W}$ motif that is located 14 amino acids downstream of the RR motif of NapF. The derivatives generated were pAN111, encoding the substitution P24A, pAN112 (P25A), pAN113 (W26Y) and pAN114 (P24A P25A W26Y) (Table 1). All of the JCB4142 transformants with these plasmids grew at the same rate as JCB4142 transformed with pAN101, and grew slightly more rapidly than the isogenic napF $F^{+}$strain JCB4141 transformed with the vector plasmid, pBR322 (Fig. 2c). It was concluded that the R-P-P-W motif, like the highly conserved double-arginine motif, is not essential for $\mathrm{NapF}$ function.

\section{In vitro and in vivo protein-protein interaction between NapF and NapA}

The cytoplasmic location of NapF is consistent with a role in maturation of NapA prior to export of the enzyme to the periplasm via the TAT pathway. To demonstrate that NapF could interact with NapA, a $\mathrm{His}_{6}-\mathrm{NapF}$ protein was constructed for use in NapA pull-down assays. The napF gene was excised from pLP802, cloned into the $\mathrm{His}_{6}$-tag vector pET16B (to generate pELHisNapF, Table 1) and expressed in E. coli BL21(DE3)pLysS. His ${ }_{6}-\mathrm{NapF}$ was purified from soluble fractions by sequential Ni-affinity, DEAE anion-exchange and S200 gel-exclusion chromatographies, followed by a second Ni-affinity step. The $\mathrm{His}_{6}{ }^{-}$ NapF protein eluted at around $480-500 \mathrm{mM}$ imidazole on 

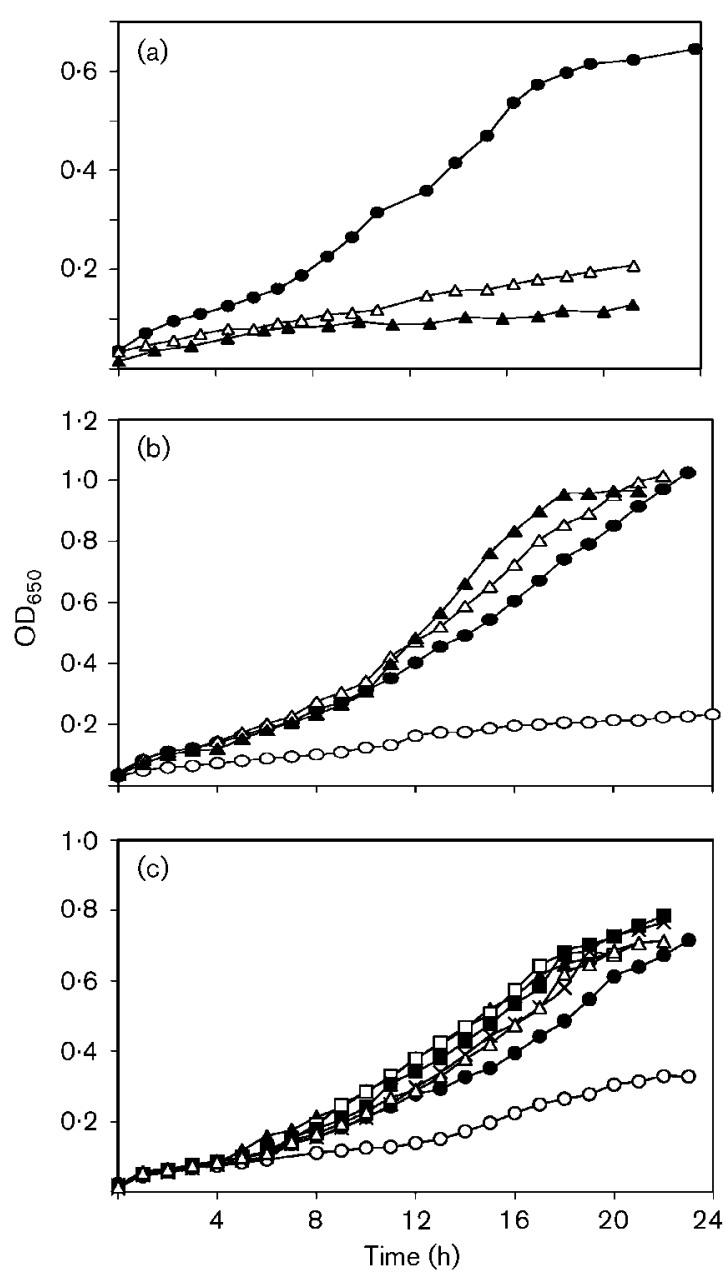

the Ni-affinity columns and was around $95 \%$ pure, as judged by Coomassie blue staining of SDS-PAGE gels, after the four steps. Tracking the NapF-containing fractions by UV-visible spectroscopy during purification revealed enrichment of a protein with the spectral properties of an iron-sulphur protein, as indicated by characteristic broad absorption features between 600 and $350 \mathrm{~nm}$. This absorption spectrum was consistent with previous amino acid sequence analysis that has suggested that NapF is an ironsulphur protein. However, the intensity of these bands varied from preparation to preparation and they were lost on storage of the protein at $4{ }^{\circ} \mathrm{C}$ or $-20^{\circ} \mathrm{C}$. This prevented reproducible quantification and suggested that the ironsulphur clusters bound by E. coli NapF were labile, a property that was also recently observed for the homologous NapF protein from the phototroph R. sphaeroides (OlmoMira et al., 2004). This is also consistent with a preliminary report that an $\mathrm{N}$-terminal fusion of the maltose-binding protein to NapF generated a protein that contained less than a stoichiometric quantity of iron and acid-labile sulphur in an approximately $1: 1$ ratio (Kim et al., 2003).

To assess whether E. coli NapA could bind to immobilized $\mathrm{His}_{6}-\mathrm{NapF}$, soluble fractions were prepared from E. coli
Fig. 2. Growth curve assays of NapF functionality. (a) Effect of deletion of the nap $F$ gene on Nap-dependent growth of strains producing only UQ. Strains were grown anaerobically in MS medium supplemented with $10 \%(\mathrm{v} / \mathrm{v}) \mathrm{NB}$ and glycerol in the presence or absence of nitrate. The cell densities were measured every hour for 24 h. $\boldsymbol{\Delta}$, JCB4141 (Nap ${ }^{+}$: no nitrate); -, JCB4141 (Nap ${ }^{+}$: nitrate added); $\triangle$, JCB4142 ( $\Delta$ napF: nitrate added). The data are representative of three independent experiments. (b) Growth complementation of the nap $F$ deletion strain with NapF expressed from a plasmid. Growth of strains expressing Nap as the only nitrate reductase and defective in MK synthesis. $\bullet$, pBR322 in JCB4141 $\left(\mathrm{Nap}^{+}\right)$; $\bigcirc$, pBR322 in JCB4142 $(\Delta$ nap $F) ; \triangle$, pAN101 in JCB4142 ( $\Delta$ napF+NapF); $\Delta$, pAN103 in JCB4142 $[\Delta$ napF+NapF (RR-AA)]. Strains were grown anaerobically in MS $+10 \%$ NB supplemented with glycerol, nitrate and ampicillin. The data are representative of three independent experiments. (c) Effect of amino acid substitutions in the proline motif of NapF on growth. Growth of strains expressing $\mathrm{Nap}$ as the only nitrate reductase and defective in the MK synthesis. - pBR322 in JCB4141 $\left(\mathrm{Nap}^{+}\right) ; \mathrm{O}, \mathrm{pBR} 322$ in JCB4142 ( $\mathrm{N}$ napF); $\boldsymbol{\Delta}$, pAN101 in JCB4142 ( napF+plasmid-encoded NapF); $\square, \quad$ pAN111 (encoding NapF P24A) in JCB4142; $\mathbf{\square}$, pAN112 (NapF P25A) in JCB4142; $\times$, pAN113 (NapF W26Y) in JCB4142; $\triangle$, pAN114 (NapF P24A:P25A:W26Y) in JCB4142. Transformants were grown anaerobically in minimal salts medium supplemented with $10 \%(\mathrm{v} / \mathrm{v}) \mathrm{NB}$, glycerol, nitrate and ampicillin. The data are representative of two independent growth experiments. Note the linear rather than exponential $y$ axis, which, because the exponential phase of growth was rather short, was used to emphasize the effects of nitrate and nap $F$ mutations on growth.

grown anaerobically on a glycerol/nitrate minimal medium and applied to Ni-affinity columns that were either charged with $\mathrm{His}_{6}$-NapF or left uncharged. Column eluate fractions were subjected to SDS-PAGE and Western blotting and then probed with anti-NapA antibodies. When soluble extracts were added to uncharged columns all of the NapA present in the soluble extract was eluted in the column wash; no binding of NapA to the column was detectable. However, when soluble extracts were applied to columns charged with $\mathrm{His}_{6}$-NapF virtually no NapA eluted in the wash buffers. Almost all of the NapA in the soluble fractions bound to the column. This established that the immobilized NapF was retaining NapA on the Ni-affinity column. Following application of an imidazole elution gradient, the NapA remained tightly bound to the column until finally eluting at 480-500 mM imidazole, which coincided with the point of NapF elution.

The NapA in these subcellular fractions is present as the mature, folded and active periplasmic enzyme with all of the cofactors bound, and this is the form of NapA pulled down by NapF in these in vitro experiments. However, this periplasmic form of NapA is unlikely to be the form with which NapF interacts initially in the cytoplasm. Thus, whilst these experiments confirmed that NapF can interact with 
folded NapA, other approaches were required to demonstrate that an interaction in the cytoplasm was possible. To this end a bacterial two-hybrid system was adopted. In this system, pairs of compatible plasmids are used to clone fragments of the gene for $B$. pertussis adenylate cyclase inframe to the $5^{\prime}$ or $3^{\prime}$ end of the coding regions of the proteins to be investigated (Karimova et al., 1998, 2000; Ladant \& Ullmann, 1999). Interaction between two test proteins, or between a test protein and itself expressed from a second plasmid, results in adenylate cyclase activity that is revealed by activation of lac $Z$ expression. Every combination of napA, $C, D, F, G$ and $H$ genes was tested in this system (Table 4). No interaction between NapF and either NapG or $\mathrm{NapH}$ was detected, consistent with our previous proposal that NapG and $\mathrm{NapH}$ form an ubiquinol dehydrogenase complex that transfers electrons from ubiquinol in the membrane to the periplasmic NapC. However, NapA apparently forms dimers or higher homopolymers, as revealed by high $\beta$-galactosidase activities when napA was cloned into two compatible pairs of plasmids. The selfassociation of $\mathrm{NapC}$ and its interaction with $\mathrm{NapH}$ were reported previously from a more qualitative plate-based assay (Brondijk et al., 2004), as were the absence of interactions between the periplasmic NapG and other components of the Nap pathway (Brondijk et al., 2004), and these are confirmed more quantitatively in the present set of analyses (Table 4). Although NapD and NapF are the only two Nap polypeptides that are located in the cytoplasm, no interactions between them were detected and neither NapD nor NapF appears to self-associate (Table 4). However, despite the fact that NapA is targeted by the TAT pathway to the periplasm, the two-hybrid assay suggests that both NapD and NapF interact strongly with NapA. As the twohybrid system only detects interactions that occur in the cytoplasm or on the cytoplasmic side of the membrane, it is inferred that NapA forms a transient complex with NapD and NapF in the cytoplasm before it is secreted as a mature enzyme by the TAT pathway.

\section{DISCUSSION}

The role of NapF in the periplasmic nitrate reductase system of bacteria has provided something of a conundrum. In E. coli, in the absence of the membrane-bound nitrate reductases NarA and NarZ, NapA provides the only pathway for respiratory nitrate reduction. NapA is then essential for growth on non-fermentable carbon sources when nitrate is the only electron acceptor available (Brondijk et al., 2002). Deletion of NapF lowers this growth rate, but does not abolish growth. However, NapF is absolutely essential for NapA-dependent growth of a strain that is defective in MK synthesis and therefore dependent on ubiquinone (UQ) for growth. The data presented in this paper show that E. coli NapF, like R. sphaeroides NapF, is located in the cytoplasm, where it can interact with NapA. Our study also provides the first direct evidence for an interaction in the cytoplasm not only between NapA and NapF, but also between NapA and NapD, which has previously been inferred (Berks et al., 1995a; Reyes et al., 1998; Potter \& Cole, 1999) but never actually demonstrated. The detailed molecular and kinetic nature of the NapF-A and NapD-A interactions remain the subject of future work, but the interaction demonstrated here suggests a role for E. coli NapF, together with NapD, in the post-translational modification of NapA prior to its export to the periplasm via the TAT pathway. A possible clue to these functions is that both an iron-sulphur centre and Mo-bis-MGD cofactor must be incorporated into NapA before it is translocated via the TAT pathway and it is

Table 4. $\beta$-Galactosidase activities from Nap interactions using the bacterial two-hybrid assay

The $\beta$-galactosidase activities were assayed as described in Methods. The unit of activity is nmol $\min ^{-1}$ ( $\mathrm{mg}_{\text {dry }}$ weight) ${ }^{-1}$. The data are means \pm SD $(n=3)$. Positive interactions are indicated in bold.

\begin{tabular}{|c|c|c|c|c|c|c|c|}
\hline Protein & Plasmid & $\frac{\text { T25 : : NapC }}{\text { pKT25-C }}$ & $\frac{\text { T25 : : NapG }}{\text { pKT25-G }}$ & $\frac{\text { T25 : : NapH }}{\text { pKT25-H }}$ & $\frac{\text { T25 : : NapF }}{\text { pKT25-F }}$ & $\frac{\text { T25 : : NapD }}{\text { pKT25-D }}$ & $\frac{\text { T25 : : NapA }}{\text { pKT25-A }}$ \\
\hline T18: : NapC & pUT18C-C & $1820( \pm 175)$ & $110( \pm 4)$ & $180( \pm 5)$ & $112( \pm 2)$ & $87( \pm 3)$ & $80( \pm 3)$ \\
\hline NapG : : T18 & pUT18-G & $97( \pm 6)$ & $88( \pm 2)$ & $100( \pm 5)$ & $41( \pm 4)$ & $88( \pm 2)$ & $91( \pm 3)$ \\
\hline T18:: NapG & pUT18C-G & $99( \pm 15)$ & $82( \pm 2)$ & $92( \pm 4)$ & $45( \pm 9)$ & $85( \pm 4)$ & $84( \pm 3)$ \\
\hline NapF:: T18 & pUT18-F & $116( \pm 0 \cdot 4)$ & $51( \pm 3)$ & $85( \pm 2)$ & $38( \pm 1)$ & $139( \pm 19)$ & $2130( \pm 652)$ \\
\hline T18::NapF & pUT18C-F & $112( \pm 1)$ & $51( \pm 2)$ & $84( \pm 1)$ & $42( \pm 10)$ & $142( \pm 7)$ & $2570( \pm 1430)$ \\
\hline NapD : : T18 & pUT18-D & $89( \pm 5)$ & $78( \pm 4)$ & $106( \pm 2)$ & $146( \pm 4)$ & $144( \pm 10)$ & $4200( \pm 690)$ \\
\hline T18::NapD & pUT18C-D & $88( \pm 4)$ & $83( \pm 5)$ & $97( \pm 4)$ & $161( \pm 3)$ & $139( \pm 18)$ & $3110( \pm 380)$ \\
\hline NapA:: T18 & pUT18-A & $87( \pm 7)$ & $86( \pm 1)$ & $81( \pm 9)$ & $6320( \pm 355)$ & $7110( \pm 570)$ & $5300( \pm 1100)$ \\
\hline
\end{tabular}


therefore possible that NapF facilitates the insertion of either one of these cofactors. In this respect the data presented here have some resonance with recent work with $R$. sphaeroides NapF that also suggested the presence of labile ironsulphur clusters and showed that addition of NapF in vitro to inactive NapA, deficient in its iron-sulphur centre, reactivated the enzyme in the presence of cysteine and cysteine desulphydrase (Olmo-Mira et al., 2004).

If $\mathrm{NapF}$ is involved in the maturation of NapA in the cytoplasm, how can we rationalize the different phenotypes of napF mutants amongst bacterial species and the absence of napF from the nap operons of some species? This is the conundrum: napF is absent from the nap operon of Paracoccus denitrificans (Berks et al., 1995b), but present in the nap operon of the closely related $R$. sphaeroides, where it is essential for the synthesis of active NapA (Reyes et al., 1998). However, as discussed above, in E. coli deletion of $n a p F$ only slows down adaptation from aerobic to anaerobic NapA-dependent growth of a $\mathrm{UQ}^{+} / \mathrm{MK}^{+}$strain (Brondijk et al., 2002), but is essential for NapA-dependent growth in a strain defective in MK synthesis (Brondijk et al., 2004 and this study). In E. coli, during aerobic growth, UQ is the most abundant quinone pool (Unden, 1988; Soballe \& Poole, 1999) and the nap operon is not expressed. Although electron transfer through the MK pool to the Nap complex in anaerobically adapted $E$. coli is much more rapid than through the UQ pool (Brondijk et al., 2002, 2004), the opposite is likely to be true during the initial stages of adaptation, before adequate NapA and MK have accumulated (Brondijk et al., 2002, 2004). Therefore any defect in NapA maturation is likely to be more apparent during this adaptation phase. These suggestions imply that, although $\mathrm{NapF}$ accelerates post-translational maturation of NapA, it is not strictly essential in E. coli. Possibly its function can be duplicated less efficiently by a component of another anaerobically expressed pathway, for example by a protein that, like NapF, is involved in cofactor insertion into the preapo-protein. This might also then be true in $P$. denitrificans, where there is no napF gene in the nap operon, but not in $R$. sphaeroides, where the napF of the nap operon is clearly essential (Reyes et al., 1998).

\section{ACKNOWLEDGEMENTS}

This study was funded by the UK Biotechnology and Biological Sciences Research Council (BBSRC) Project Grants 6/P11528 and P21080, by BBSRC studentship 01B1C07137 to E. R. L. and by a Training Scholarship from the Royal Thai Government to A. N.

\section{REFERENCES}

Berks, B. C., Ferguson, S. J., Moir, J. W. \& Richardson, D. J. (1995a). Enzymes and associated electron transport systems that catalyse the respiratory reduction of nitrogen oxides and oxyanions. Biochim Biophys Acta 1232, 97-173.

Berks, B. C., Richardson, D. J., Reilly, A. R., Willis, A. \& Ferguson, S. J. (1995b). The napEDABC gene cluster encoding the periplasmic nitrate reductase system of Thiosphaera pantotropha. Biochem J 309, 983-992.

Berks, B. C., Sargent, F. \& Palmer, T. (2000). The Tat protein export pathway. Mol Microbiol 35, 260-274.

Blasco, F., lobbi, C., Ratouchniak, J., Bonnefoy, V. \& Chippaux, M. (1990). Nitrate reductases of Escherichia coli: sequence of the second nitrate reductase and comparison with that encoded by the narGHJI operon. Mol Gen Genet 222, 104-111.

Bolivar, F., Rodriguez, R. L., Greene, P. J., Betlach, M. C., Heyneke, N. L., Boyer, H. W., Crosa, J. H. \& Falkow, S. (1977). Construction and characterization of new cloning vehicles. II. A multipurpose cloning system. Gene 2, 95-113.

Brondijk, T. H., Fiegen, D., Richardson, D. J. \& Cole, J. A. (2002). Roles of NapF, NapG and NapH, subunits of the Escherichia coli periplasmic nitrate reductase, in ubiquinol oxidation. Mol Microbiol 44, 245-255.

Brondijk, T. H. C., Nilavongse, A., Filenko, N., Richardson, D. J. \& Cole, J. A. (2004). The NapGH components of the periplasmic nitrate reductase of Escherichia coli K-12: location, topology, and physiological roles in quinol oxidation and redox balancing. Biochem J 379, 47-55.

Clegg, S. J., Jia, W. \& Cole, J. A. (2006). Role of the Escherichia coli nitrate transport protein, NarU, during severe nutrient starvation and slow growth. Microbiology 152, 2091-2100.

Grove, J., Tanapongpipat, S., Thomas, G., Griffiths, L., Crooke, H. \& Cole, J. (1996). Escherichia coli K-12 genes essential for the synthesis of $c$-type cytochromes and a third nitrate reductase located in the periplasm. Mol Microbiol 19, 476-481.

lobbi, C., Santini, C. L., Bonnefoy, V. \& Giordano, G. (1987). Biochemical and immunological evidence for a second nitrate reductase in Escherichia coli K12. Eur J Biochem 168, 451-459.

lobbi-Nivol, C., Santini, C. L., Blasco, F. \& Giordano, G. (1990). Purification and further characterization of the second nitrate reductase of Escherichia coli K12. Eur J Biochem 188, 679-687.

Jayaraman, P.-S., Peakman, T. C., Busby, S. J. W., Quincey, R. V. \& Cole, J. A. (1987). Location and sequence of the promoter of the gene for the NADH-dependent nitrite reductase of Escherichia coli and its regulation by oxygen, the Fnr protein and nitrite. J Mol Biol 196, 781-788.

Karimova, G., Pidoux, J., Ullmann, A. \& Ladant, D. (1998). A bacterial two-hybrid system based on a reconstituted signal transduction pathway. Proc Natl Acad Sci U S A 95, 5752-5756.

Karimova, G., Ullmann, A. \& Ladant, D. (2000). A bacterial twohybrid system that exploits a cAMP signaling cascade in Escherichia coli. Methods Enzymol 328, 59-73.

Kim, H. R., Lee, Y. C., Won, J. S. \& Choe, M. H. (2003). AAS and ICP-AES analysis of the iron-sulfur cluster in YojG (NapF) protein of aeg-46.5 operon in Escherichia coli. Bull Korean Chem Soc 24, 1849-1852.

Ladant, D. \& Ullmann, A. (1999). Bordetella pertussis adenylate cyclase: a toxin with multiple talents. Trends Microbiol 7, 172-176.

Manoil, C. (1991). Analysis of membrane protein topology using alkaline phosphatase and beta-galactosidase gene fusions. Methods Cell Biol 34, 61-75.

Minton, N. P. (1984). Improved plasmid vectors for the isolation of translational lac gene fusions. Gene 31, 269-273.

Olmo-Mira, F., Richardson, D. J., Castillo, F., Moreno-Vivian, C. \& Roldan, D. (2004). NapF is a cytoplasmic iron-sulfur protein required for $\mathrm{Fe}-\mathrm{S}$ cluster assembly in the periplasmic nitrate reductase. J Biol Chem 279, 49727-49735.

Paulsen, I. T., Brown, M. H., Dunstan, S. J. \& Skurray, R. A. (1995). Molecular characterization of the staphylococcal multidrug resistance export protein QacC. J Bacteriol 177, 2827-2833. 
Pope, N. R. \& Cole, J. A. (1984). Pyruvate and ethanol as electron donors for nitrite reduction by Escherichia coli K12. J Gen Microbiol 130, 1279-1284.

Potter, L. C. \& Cole, J. A. (1999). Essential roles for the products of the napABCD genes, but not napFGH, in periplasmic nitrate reduction by Escherichia coli K-12. Biochem J 344, 69-76.

Potter, L. C., Millington, P., Griffiths, L., Thomas, G. H. \& Cole, J. A. (1999). Competition between Escherichia coli strains expressing either a periplasmic or a membrane-bound nitrate reductase: does Nap confer a selective advantage during nitrate-limited growth? Biochem J 344, 77-84.

Reyes, F., Roldan, M. D., Klipp, W., Castillo, F. \& Moreno-Vivian, C. (1996). Isolation of periplasmic nitrate reductase genes from Rhodobacter sphaeroides DSM 158: structural and functional differences among prokaryotic nitrate reductases. Mol Microbiol 19, 1307-1318.

Reyes, F., Gavira, M., Castillo, F. \& Moreno-Vivian, C. (1998). Periplasmic nitrate-reducing system of the phototrophic bacterium Rhodobacter sphaeroides DSM 158: transcriptional and mutational analysis of the napKEFDABC gene cluster. Biochem $J$ 331, 897-904.

Richardson, D. J. \& Ferguson, S. J. (1992). The influence of carbon substrate on the activity of the periplasmic nitrate reductase in aerobically grown Thiospaera pantotropha. Arch Microbiol 157, 535-537.

Santini, C. L., Ize, B., Chanal, A., Muller, M., Giordano, G. \& Wu, L. F. (1998). A novel Sec-independent periplasmic protein translocation pathway in Escherichia coli. EMBO J 17, 101-112.

Sears, H. J., Sawers, G., Berks, B. C., Ferguson, S. J. \& Richardson, D. J. (2000). Control of periplasmic nitrate reductase gene expression
(napEDABC) from Paracoccus pantotrophus in response to oxygen and carbon substrates. Microbiology 146, 2977-2985.

Simon, J., Sanger, M., Schuster, S. C. \& Cross, R. (2003). Electron transport to periplasmic nitrate reductase (NapA) of Wolinella succinogenes is independent of a NapC protein. Mol Microbiol 49, 69-75.

Soballe, B. \& Poole, R. K. (1999). Microbial ubiquinones: multiple roles in respiration, gene regulation and oxidative stress management. Microbiology 145, 1817-1830.

Stanley, N. R., Sargent, F., Buchanan, G., Shi, J., Stewart, V., Palmer, T. \& Berks, B. C. (2002). Behaviour of topological marker proteins targeted to the Tat protein transport pathway. Mol Microbiol 43, 1005-1021.

Stewart, V. (1988). Nitrate respiration in relation to facultative metabolism in enterobacteria. Microbiol Rev 52, 190-232.

Stewart, V., Lu, Y. \& Darwin, A. J. (2002). Periplasmic nitrate reductase (NapABC enzyme) supports anaerobic respiration in Escherichia coli K-12. J Bacteriol 184, 1314-1323.

Unden, G. (1988). Differential roles for menaquinone and demethylmenaquinone in anaerobic electron transport of E. coli and their fnr-independent expression. Arch Microbiol 150, 499-503.

Wang, H., Tseng, C. P. \& Gunsalus, R. P. (1999). The napF and nar $G$ nitrate reductase operons in Escherichia coli are differentially expressed in response to submicromolar concentrations of nitrate but not nitrite. J Bacteriol 184, 5303-5308.

Weiner, J. H., Bilous, P. T., Shaw, G. M., Lubitz, S. P., Frost, L., Thomas, G. H., Cole, J. A. \& Turner, R. J. (1998). A novel and ubiquitous system for membrane targeting and secretion of cofactorcontaining proteins. Cell 93, 93-101. 